\title{
Positron Range Modeling for Statistical PET Image Reconstruction
}

\author{
${ }^{\dagger}$ Bing Bai, ${ }^{\ddagger}$ Ananya Ruangma, ${ }^{\ddagger}$ Richard Laforest, ${ }^{\ddagger}$ Yuan-Chuan Tai and ${ }^{\dagger}$ Richard M. Leahy \\ ${ }^{\dagger}$ Signal and Image Processing Institute, University of Southern California, Los Angeles, CA 90089 \\ ${ }^{\ddagger}$ School of Medicine, Washington University, St. Louis, MO, 63110
}

\begin{abstract}
Positron range is one of the factors that fundamentally limits the spatial resolution of PET images. With the higher resolution of small animal imaging systems and increased interest in using higher energy positron emitters, it is important to consider range effects when designing image reconstruction methods. The positron range distribution can be measured experimentally or calculated using approximate analytic formulae or Monte Carlo simulations. We investigate the use of this distribution within a MAP image reconstruction framework. Positron range is modeled as a blurring kernel and included as part of the forward projection matrix. We describe the use of a 3D isotropic shift-invariant blur kernel, which assumes that positrons are propagating in a homogeneous medium and is computed by Monte Carlo simulation using EGS4. We also propose a new shift-variant blurring model for positron range that accounts for spatial inhomogeneities in the positron scatter properties of the medium. Monte Carlo simulations, phantom, and animal studies with the isotopes $\mathrm{Cu}-60$ and $\mathrm{Cu}-64$ are presented.
\end{abstract}

\section{INTRODUCTION}

Positron range is one of the factors that fundamentally limits the spatial resolution of PET images [1]. A positron travels a short distance before positron-electron annihilation. The range of the positron depends on its energy as well as the effective atomic number and atomic weight of the medium. Major interactions between positrons and the surrounding medium include Coulomb elastic collisions with atomic nuclei and inelastic collisions with atomic electrons [1]. In most cases a positron first loses all its energy and then annihilates with an electron [2]. In each inelastic collision, the positron only loses a small part of its energy [1], as a result many collisions will happen before annihilation and the trajectory of each positron is tortuous.

Positron range in water has been measured experimentaly for several medically important isotopes [3], [4], [5]. These results show considerable variation, primarily because the resolution of the detectors were comparable to the positron range. Palmer and Brownell [6] proposed a 3D-Gaussian model for the annihilation point distribution, assuming the positrons behave diffusively. Their calculation is based on an empirical range-energy formula. Difficulties in experimental range measurements have also lead to the recent use of Monte Carlo simulation to calculate positron range [1], [7].

This work was supported in part by Grant R01 EB000363 from the National Institute of Biomedical Imaging and Bioengineering.
The effect of positron range is a blurring of the reconstructed image. Based on the measured positron annihilation point distribution, Derenzo and Haber proposed a method to remove the blurring by spatial deconvolution [8], [9]. While this method can partly recover the resolution loss, by decoupling the deconvolution from image reconstruction we lose the ability to optimally handle noise amplification through the use of an accurate likelihood function.

We have developed a 3D MAP reconstruction method in which a factored system model is used [10]. In our previous 3D MAP reconstructions, positron range has been ignored. Recently, the development of new detector technology has reduced crystal size so that $1 \mathrm{~mm}$ spatial resolution is potentially achievable with small animal PET scanners such as the microPET II [11]. The spatial resolution of these scanners is comparable to the positron range of the isotopes that are commonly used (e.g., the mean positron range of F-18 in water is $0.5 \mathrm{~mm}$ ). High-energy isotopes with longer positron range have also been used in small animal PET studies [7]. In this paper we describe positron range modeling in our system model using blurring operators in the image space. We describe two approaches. The first uses a shift-invariant blurring operator that implicitly assumes homogeneous range throughout the subject. The second approach uses a sequence of convolutions to account for the effects of inhomogeneities in the subject. Preliminary results using high-energy isotopes show significant improvements in the spatial resolution of the reconstructed images compared with methods without positron range modeling.

\section{MAP ImAGe ReCONSTRUCTION}

Maximum a Posteriori (MAP) image reconstruction is a Bayesian approach that can combine accurate statistical and physical models for the data with a prior on the unknown image [10]. PET data are modeled as a collection of independent Poisson random variables with mean

$$
\overline{\mathbf{y}}=\mathbf{P x}+\overline{\mathbf{r}}+\overline{\mathbf{s}}
$$

where $\overline{\mathbf{r}}$ is the mean of the randoms, and $\overline{\mathbf{s}}$ is the mean of the scattered events. $\mathbf{P}$ is the system matrix describing the probability that an unscattered event is detected, which we factor as [10]

$$
\mathbf{P}=\mathbf{P}_{\text {norm }} \mathbf{P}_{\text {blur }} \mathbf{P}_{\text {attn }} \mathbf{P}_{\text {geom }} \mathbf{P}_{\text {range }}
$$


TABLE I

MEAN POSITRON RANGE IN WATER FOR ISOTOPES OF INTEREST IN PET

\begin{tabular}{|c|c|c|c|}
\hline Isotope & Mean range $(\mathrm{mm})$ & Isotope & Mean range $(\mathrm{mm})$ \\
\hline $\mathrm{F}-18$ & 0.51 & Cu-60 & 3.09 \\
$\mathrm{Cu}-64$ & 0.57 & Br-76 & 3.47 \\
$\mathrm{~N}-13$ & 1.31 & $\mathrm{Cu}-62$ & 4.39 \\
$\mathrm{Cu}-61$ & 1.32 & Ga-66 & 6.13 \\
\hline
\end{tabular}

where $\mathbf{P}_{\text {range }}$ is a local, shift variant blurring operator applied in the image space that models the positron range effect; $\mathbf{P}_{\text {geom }}$ is the geometric projection matrix describing the probability that a photon pair reaches the front faces of a detector pair in the absence of attenuation and assuming perfect photon pair collinearity; $\mathbf{P}_{\text {blur }}$ is a blurring operator applied in sinogram space that models photon pair non-collinearity, intercrystal scatter and crystal penetration; $\mathbf{P}_{\text {attn }}$ is a diagonal matrix containing attenuation correction factors for each detector pair; and $\mathbf{P}_{\text {norm }}$ is a diagonal matrix containing the normalization factors for unscattered events.

In this paper we propose a convolutional model for the effects of positron range which we incorporate in the MAP reconstruction procedure through the factor $\mathbf{P}_{\text {range }}$. Our goal is to accurately model this effect in a computationally efficient manner.

\section{Positron Range Model}

\section{A. Homogeneous Medium}

Since positrons are emitted isotropically, it is reasonable to assume that the annihilation point distribution in a homogeneous medium is also isotropic. We model the annihilation points in an infinite homogeneous medium with a threedimensional isotropic density function centered at the origin. The shape of the density function was determined by a Monte Carlo simulation using EGS4 [7].

The annihilation point probability density function (PDF) $f(x, y, z)$ represents the probability of a positron emitted from the origin annihilating at point $(x, y, z)$. The PDF for a homogeneous medium is isotropic, so that is only a function of the distance from the origin $r=\sqrt{x^{2}+y^{2}+z^{2}}$, i.e. $f(x, y, z)=f(r)$. Using spherical coordinates $(r, \theta, \phi)$ and integrating over $\theta$ and $\phi$, gives the one dimensional PDF $F(r)$ for annihilation at a distance $r$ from the origin:

$$
F(r)=4 \pi r^{2} f(r)
$$

The 1D $(F(r))$ and 3D $(f(r))$ annihilation PDFs in water, computed for several isotopes using EGS4, are shown in Figure 1. Table I lists the mean range for each of these isotopes.

The simplest approach to modeling positron range is to assume the subject is homogeneous and model the effect as a shift-invariant blurring function formed by the 3D PDF $f(r)$ computed for soft tissue. This can be directly incorporated in the system model, equation (2), as a discrete convolution operator or blur kernel in $\mathbf{P}_{\text {range }}$. To compute this blur kernel we need to discretize the distribution $f(r)$. To do this we divide
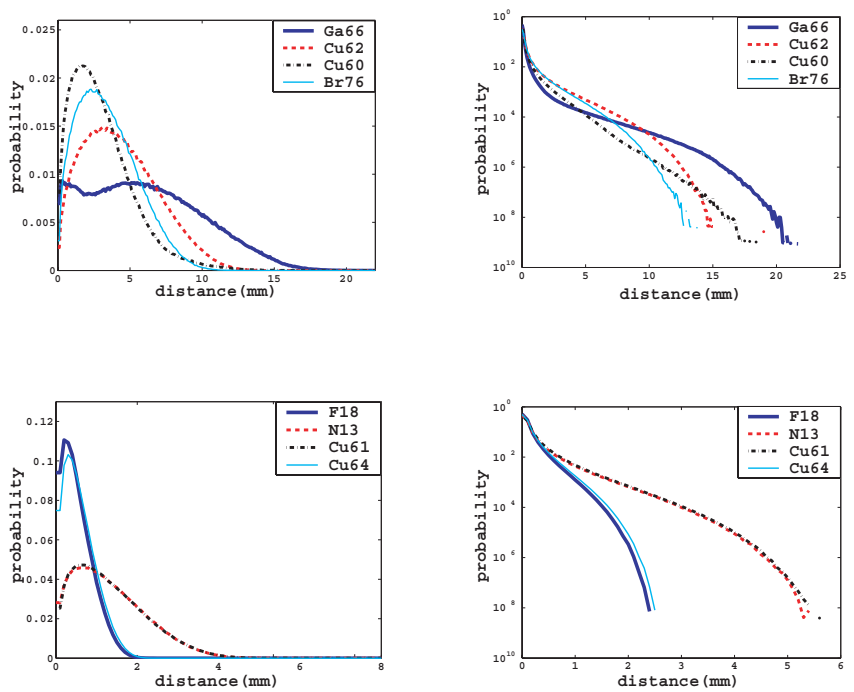

Fig. 1. Left column: 1D annihilation PDF, $F(r)$; Right column: 3D annihilation PDF, $f(r)$, for several medically important isotopes from Monte Carlo simulation.

each voxel into sub-voxels, the probability that an annihilation happens in a sub-voxel is approximated by $f\left(r_{i}\right) \delta$, where $r_{i}$ is the distance from the center of $i^{\text {th }}$ sub-voxel to the origin and $\delta$ is the volume of the sub-voxel. The probability that an annihilation occurs in a given voxel is the sum of the probabilities in its sub-voxels.

In the following subsection we describe a more accurate model that takes into account inhomogeneities in the subject. However, we first note that a simple but effective modification to the homogeneous model is to truncate the blur kernels at the boundary of the subject. The approximate effect of this truncation is to assume that any positrons that leave the body escape and do not contribute photon pairs to the sinogram data. This method requires only that the boundary of the subject be determined either from a coregistered CT scan or a reconstructed transmission image. Implementation of this correction is straightforward since we need only window the convolutions with the support of the subject when computing forward and back projections.

\section{B. Inhomogeneous Medium}

In real subjects, positron scatter is neither homogeneous nor isotropic but depends on the surrounding medium. A positron has much higher probability of traveling a long distance before annihilation in air than in the water. The isotropic model described above is accurate only when the medium is homogeneous. When the medium is inhomogeneous with arbitrary geometry, no analytical model is available. The best way to calculate positron range accurately in this case is through Monte Carlo simulation. This is not practical for image reconstruction purposes. Therefore, here we propose a new shift-variant blurring model that approximates the transport and annihilation of positrons in a dense medium. This is achieved 


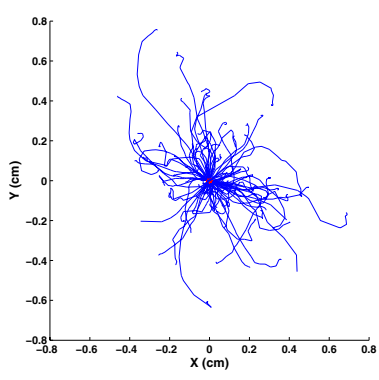

Fig. 2. Trajectories of 100 positrons emitted from a $\mathrm{Cu}-60$ point source in water, simulated using EGS4.

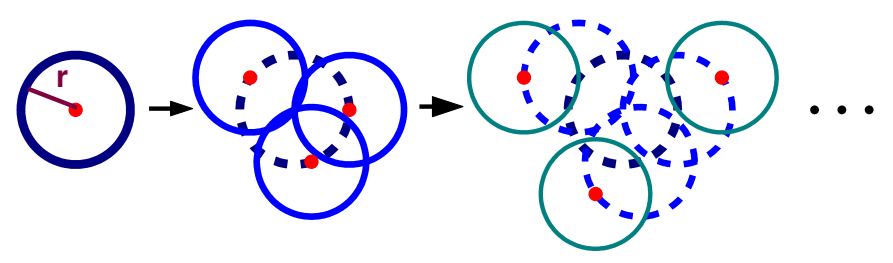

Fig. 3. Illustration of the inhomogeneous positron range model.

using a sequence of pairs of isotropic convolutions where one convolution tracks the positrons annihilated at each stage, and the other tracks the surviving positrons. The local convolution kernels are determined by the tissue type (lung, soft tissue, bone) surrounding each voxel.

Figure 2 shows a Monte Carlo simulation of the trajectories of 100 positrons emitted from a $\mathrm{Cu}-60$ point source in water. We can split these trajectories into two groups: those that never leave a sphere of radius $r$ and annihilate within the sphere, and those that do leave the sphere. We can model this process by generating two images: the first image gives the spatial distribution of positron annihilation points within the sphere. The second is an image of surviving positrons that are confined to the surface of the radius $r$ sphere, with a uniform distribution on that surface. This process is illustrated in Figure 3. We can then track the fate of these surviving photons as follows. Each point on the sphere surface is viewed as a secondary source. The positrons from this secondary source either remain within a sphere of radius $r$, centered at that point, and annihilate within the sphere, or they leave the sphere. Again, we can produce two images. The first is the distribution of positron annihilations confined to the set of spheres defined for the set of secondary sources. The second image is that of the surviving photons that again are confined to the surfaces of spheres of radius $r$, centered at the locations of each of the secondary sources. This procedure is repeated until all positrons are annihilated or escape the subject.

We can use the positron trajectories from a Monte Carlo simulation of a point source to determine the distribution of positrons that are annihilated and that survive at each stage of this procedure. Note that the two images that are formed at each stage of this procedure are both convolutions of the results of the previous stage. The first (the annihilation image) is a convolution of the previous image of surviving positrons with
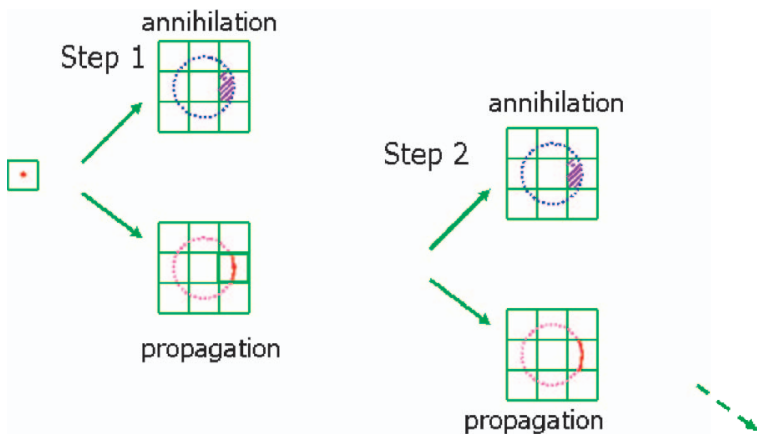

Fig. 4. Illustration of the convolutions in each stage of the inhomogeneous range model.

a blur function which is zero outside the sphere of radius $r$ and equal to the annihilation density within the sphere. The second image (the surviving or propagating positrons) is formed as the convolution of the previous image of surviving positrons with a kernel that is nonzero only on a spherical surface of radius $r$, with a value equal to the fraction of surviving positrons in that stage normalized by the surface area of the sphere. This process is illustrated in Figure 4. Note that at each stage, the surviving positrons will have lower average energy than in the previous stage, so that the probability of annihilation within each stage increases as we move from one stage to the next. Note also, that at each stage we record the annihilation of only those positrons whose trajectories do not leave the sphere of radius $r$; it is possible for other positrons to subsequently annihilate within this sphere, but if they first leave the sphere, then these annihilations are accounted for at a later stage. This property is important since it allows us to model the effect of inhomogeneities within the subject as we describe below.

Note that if the positron source is spatially distributed rather than a point source, the above procedure can still be used by applying the convolution operators throughout the source volume. The procedure can also be readily discretized into a set of discrete convolutions on the image volume. Since the convolutions are all local, the approach is a practical alternative to Monte Carlo simulation, and can be implemented as a linear operator on the source distribution, which we represent as $\mathbf{P}_{\text {range }}$ in the system model in equation (2).

This procedure of successive convolutions will track the fate of all positrons within the subject. It implicitly involves two assumptions that we now describe and discuss.

- We assume that positrons are scattered isotropically. While this is true after several scatters, it is not the case for the first few scattering events. This inevitably causes errors in our range model which we minimize by fitting the parameters of the discrete convolution model directly to the $3 \mathrm{D}$ range function $f(r)$ rather than using the parameters determined directly from the Monte Carlo positron trajectories. We illustrate the accuracy of this approximation in the following section.

- The image is assumed to be locally homogeneous. Using Monte Carlo simulations we can determine the convolution 
kernels for each tissue type of interest; here we normally assume the body consists of soft tissue, lung and bone. The kernels are then applied to each voxel in turn to perform the convolution. Since we discretize the volume, we must assume a single tissue type at each voxel in order to select the appropriate convolution kernel. If we select the sphere radius $r$ equal to the voxel size and assume the mass of the voxel is concentrated at the center of each voxel, selection of a kernel for a specific tissue type is equivalent to assuming each voxel is homogeneous, but that neighboring voxels can be of different tissue types. Consequently, the local homogeneity assumption only applies at the subvoxel level and is therefore not restrictive.

To discretize this process, we use two $3 \times 3 \times 3$ blur kernels at each stage, one for annihilation and the other for propagation. These kernels are calculated by discretizing the continuous model described above for each medium. In forward and back projection, the kernels used for each voxel should be determined from a co-registered CT image segmented into bone, soft tissue and lung regions. Note that at each stage, the kernels at a particular voxel change as the average energy of the surviving positrons reduces.

\section{RESUlTs}

\section{A. Monte Carlo Simulation of Two Media: Inhomogeneous Model}

To evaluate our inhomogeneous model, we performed a Monte Carlo simulation using two media. In the simulation a $\mathrm{Cu}-60$ point source was placed in an infinite medium composed of soft tissue and lung, separated by an infinite plane at $z=0$. The point source was moved from the boundary into the soft tissue with a $1 \mathrm{~mm}$ stepsize. We calculated the positron annihilation density distribution using the inhomogeneous model, and compared this with the distribution computed from the Monte Carlo simulation data. For display purposes, we reduced the results to one dimension by forming planar projections for each value of $z$, i.e. for each point source location we computed the projected point spread function (PPSF) as the sum of the annihilation density distribution $f(x, y, z)$ over each z plane: $\operatorname{PPSF}(z)=\sum_{x} \sum_{y} f(x, y, z)$.

Figure 5 compares PPSFs from the Monte Carlo simulation with the inhomogeneous convolution model when the point source is at the soft tissue/lung boundary and when it is $3 \mathrm{~mm}$ inside the soft tissue. The fractional mean squared error in the PPSF is also shown as a function of the location of the point source. These results demonstrate that the inhomogeneous model is able to capture the asymmetries in the annihilation distribution along the $z$ axis for this inhomogeneous medium, although the accuracy deteriorates as the boundary is approached. We note that this simulation is for $\mathrm{Cu}-60$ which has a very high positron energy and hence the isotropic scatter assumption is more strongly violated here than for lower energy positrons. Consequently we can expect lower mean squared error for isotopes of lower energy.
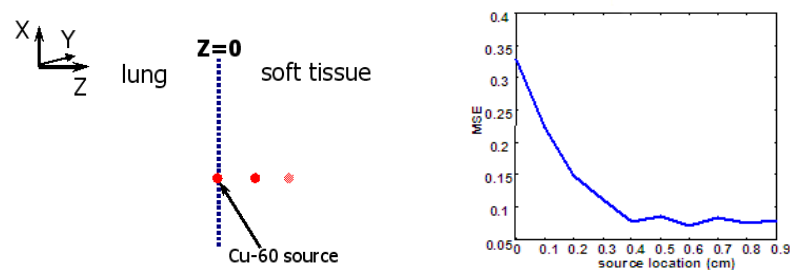

(a) Illustration of the source and media locations for study of inhomogeneity.
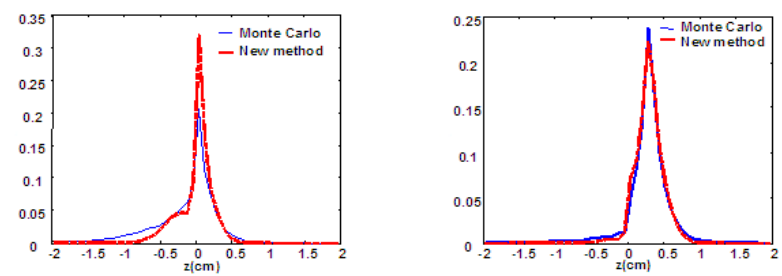

(c) PPSF for a point source at the soft tissue/lung boundary. (b) Mean squared error of PPSF as a function of point source location on $z$ axis.

(d) PPSF for a point source $3 \mathrm{~mm}$ inside the soft tissue region.

Fig. 5. Comparison of the inhomogeneous convolutional model with Monte Carlo data for a a Cu- 60 point source placed in a medium consisting of planar slabs of soft tissue and lung.

\section{B. Phantom Simulation: Homogeneous Model}

We simulated a $6 \mathrm{~cm} \times 5 \mathrm{~cm}$ 3D mouse chest phantom and the image was blurred with the positron range kernel simulated using $\mathrm{Cu}-60$ in water. The blur kernel was truncated using the true boundary of the object, then the masked image was forward projected to obtain the sinogram. The mean positron range of $\mathrm{Cu}-60$ is $3.09 \mathrm{~mm}$. The parameters of the Concorde microPET R4 rodent scanner were used in this simulation. The simulation is noiseless for the purposes of demonstrating the artifacts that arise if a homogeneous range function is used for reconstruction. Figure 6 shows the central plane of the phantom and the images reconstructed with and without truncating the blur kernel when forward and backprojecting. Artifacts are clearly seen in the image reconstructed without truncating. We see similar artifacts in in vivo mouse scans with positron range correction when we do not truncate the range function. This simulation indicates that in general a simple homogeneous model that compensates for positron range is not sufficient and will lead to artifacts, particularly at the boundaries of the subject.

\section{Animal Study}

We scanned mice injected with $\mathrm{Cu}$-DO3P, a bone-seeking agent. Three studies were performed using three different $\mathrm{Cu}$ isotopes: $\mathrm{Cu}-60, \mathrm{Cu}-61$, and $\mathrm{Cu}-64$. Data were acquired using the Concorde microPET R4 scanner. The intrinsic resolution of the R4 is less than $2 \mathrm{~mm}$. In these studies activity was primarily 


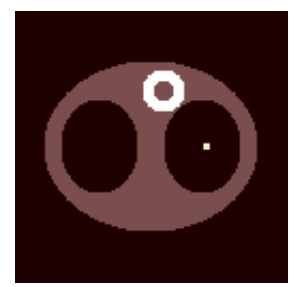

(a)

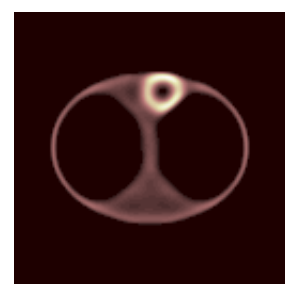

(b)

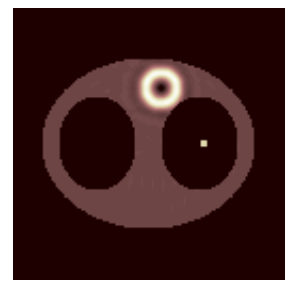

(c)
Fig. 6. Cu-60 chest phantom simulation. (a) Central plane of the phantom simulated; (b) Image reconstructed without truncating the positron range blur kernel at the object boundary; (c) Image reconstructed with truncation.

in bone and internal organs so that boundary masking was not essential.

Figure 7 shows maximum intensity projection (MIP) images for the $\mathrm{Cu}-60$ and $\mathrm{Cu}-64$ labeled tracer reconstructed using FBP, MAP with positron range modeling (homogeneous model) and MAP without positron range modeling. The effect of positron range modeling can be verified visually.

\section{CONCLUSION}

We have presented a new model for positron range that can be included as part of a statistical reconstruction method such as MAP or OSEM. The model accounts for inhomogeneities in the positron scatter properties of the subject through decomposition of the model into a sequence of local convolutions. Through Monte Carlo simulations we have verified that this model is able to capture asymmetries in positron range that occur for point sources near tissue boundaries. A full evaluation and application to in vivo data is still needed. We also show a preliminary in vivo study of correction for positron range in which the homogeneous model is used and appears to demonstrate significant improvement in resolution. However, the results of the phantom study presented indicate that these results need to be interpreted carefully, since inaccurate range correction can produce apparent resolution enhancement while actually introducing significant image artifacts. This phantom simulation demonstrates that some form of modeling of inhomogeneity in positron range is necessary when range is included in the system model.

\section{REFERENCES}

[1] C. S. Levin and E. J. Hoffman, Calculation of Positron Range and Its Effect on the Fundamental Limit of Positron Emission Tomography System Spatial Resolution, Phys. Med. Biol., 44:781-99, 1999.

[2] W. Heitler, The Quantum Theory of Radiation, pp. 268-75, New York: Dover, 1953.

[3] Z. H. Cho, J. K. Chan, L. Ericksson, M. Singh, S. Graham, N. S. Macdonald and Y. Yano, Positron Ranges Obtained From Biomedically Important Positron-Emitting Radionuclides, J. Nucl. Med., 16:1174-6, 1975.

[4] M. E. Phelps, E. J. Hoffman and S. Huang, Effects of Positron Range on Spatial Resolution, J. Nucl. Med., 16:649-52, 1975.

[5] S. E. Derenzo, Precision Measurement of Annihilation Point Spread Distributions for Medically Important Positron Emitters, Positron Annihilation R.R. Hasiguti and K. Fujiwara, Eds. Sendai, Japan: The Japan Institute of Metals , 819-23, 1979.

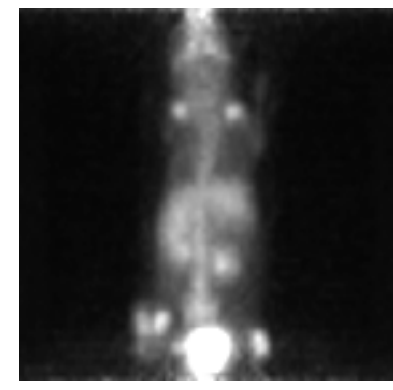

(a)

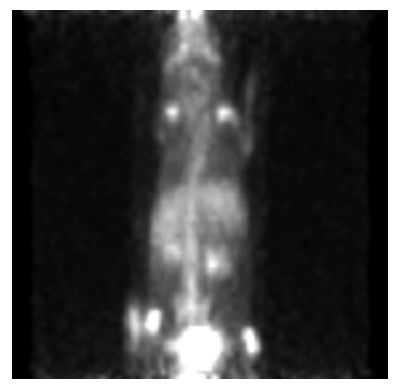

(c)

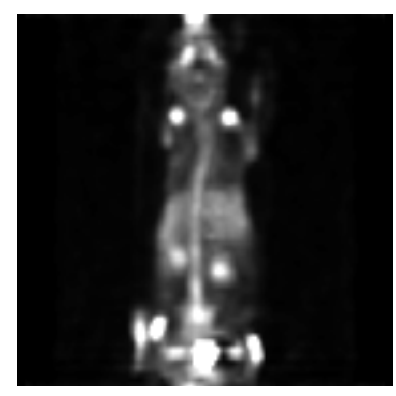

(e)

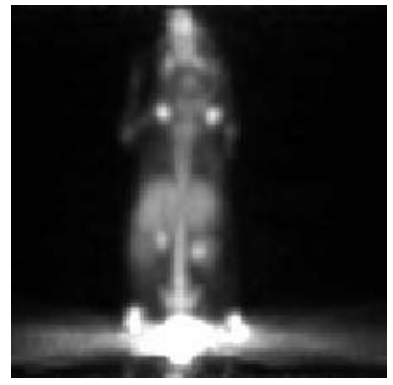

(b)

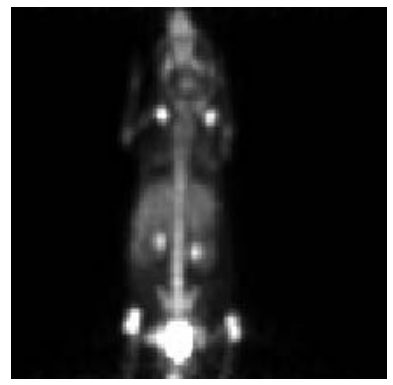

(d)

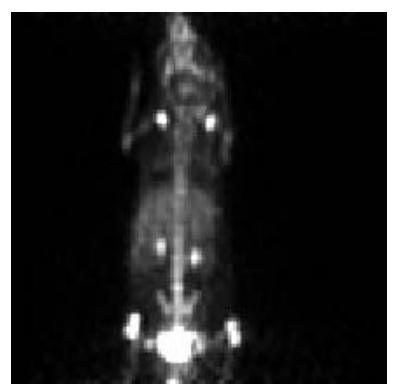

(f)
Fig. 7. MIP images of DO3P mouse scanned using (a,c,e) Cu-60 and (b,d,f) $\mathrm{Cu}-64$. Images reconstructed using $(\mathrm{a}, \mathrm{b}) \mathrm{FBP},(\mathrm{c}, \mathrm{d}) \mathrm{MAP}$ without positron range model and (e,f) MAP with positron range model.

[6] M. R. Palmer and G. L. Brownell, Annihilation Density Distribution Calculations for Medically Important Positron Emitters, IEEE Trans. Med. Imaging, 11:373-8, 1992.

[7] R. Laforest, D. J. Rowland and M. J. Welch, MicroPET Imaging with Nonconventional Isotopes, Conference Record 2001 IEEE NSS/MIC, 3:15726, 2001.

[8] S. E. Derenzo, Mathematical Removal of Positron Range Blurring in High Resolution Tomography, IEEE Trans. Nucl. Sci., 33(1):565-9, 1986.

[9] S. F. Haber, S. E. Derenzo and D. Uber, Application of Mathematical Removal of Positron Range Blurring in Positron Emission Tomography, IEEE Trans. Nucl. Sci., 37:1293-9, 1990.

[10] J. Qi, R. M. Leahy, S. R. Cherry, A. Chatziioannou and T. H. Farquhar, High resolution 3D Bayesian image reconstruction using the microPET small-animal scanner, Phys. Med. Biol., 47:1001-13, 1998.

[11] A. Chatziioannou, Y. C. Tai, N. Doshi and S. R. Cherry, Detector Development for microPET II: A $1 \mu \mathrm{L}$ Resolution ET Scanner for Small Animal Imaging, Phys. Med. Biol., 46:2899-910, 2001. 